Tohoku J. Exper. Med., 1961, 73, 230-246

\title{
The Electrocardiographic Sequelae of Right Ventriculotomy in Patients with Ventricular Septal Defect
}

By

\author{
Rikuro Sasaki \\ From the Medical Clinic of Prof. T. Ku rokawa, \\ Tohoku University, Sendai
}

(Received for publication, September 13, 1960)

Recent developments in operative techniques have made possible the surgical treatment of many varieties of congenital diseases of the heart. In fact, most of the patients operated for the closure of cardiac septal defects recuperate with surprising rapidity, and the morbidity associated with the surgery is now low. Many of the children have essentially normal hearts on both physically and X-ray examination after the immediate postoperative period, and in general they seem to thrive. This state does not mean that open heart surgery may not sometimes produce myocardial changes which are not apparent immediately. Although some ${ }^{1 / 2 / 3 / 4)}$ reported the postoperative electrocardiographic changes in patients with the atrial and ventricular septal defects and discussed a correlation between the electrocardiograms and hemodynamic changes, no one has apparently been concerned with possible ill effects of open heart surgery on the heart. The persistent electrocardiographic changes which I have observed after right ventriculotomy for repair of ventricular septal defect suggest that this procedure is not always innocuous as far as the myocardium is concerned even though the patient may be well clinically. It is still too early to evaluate fully the clinical significance of some of the abnormalities.

This paper is a comparative study of the electrocardiograms of 90 patients before and after the surgical repair of their ventricular septal defects from the standpoint of possible ill effects on the heart.

\section{MATERIAL AND METHODS}

Present study is based on the patients from the State University of Iowa Hospitals, Iowa City, Iowa, U.S.A. The series consisted of 43 males and 47 females. Their ages ranged from 2 to 36 years with an average of 9.4 , children under 16 years of age being 76 out of total 90 . Table I lists their cardiac lesions and other associated cardiac anomalies. The patients were divided into 4 major 
TaBle I. Classification of Caridac Lesions (I)

\begin{tabular}{|c|c|c|c|}
\hline Kinds of lesions & $\begin{array}{c}\text { No. of } \\
\text { cases }\end{array}$ & Kinds of lesions & $\begin{array}{l}\text { No. of } \\
\text { cases }\end{array}$ \\
\hline VSD isolated & 52 & \multirow{2}{*}{$\begin{array}{l}\text { VSD with patent ductus arterious } \\
\text { and pulmonary hypertension }\end{array}$} & \multirow{2}{*}{1} \\
\hline VSD with infundibular sterosis & 11 & & \\
\hline $\begin{array}{l}\text { VSD with infundibular stenosis } \\
\text { and valvular pulmonic stenosis }\end{array}$ & 4 & $\begin{array}{l}\text { VSD with atrial septal defect and } \\
\text { valvular pulmonic stenosis }\end{array}$ & 1 \\
\hline VSD with pulmonary hypertension & 7 & $\begin{array}{l}\text { VSD with pulmonary hypertension } \\
\text { and coarotation of the aorta. }\end{array}$ & 1 \\
\hline $\begin{array}{l}\text { VSD with pulmonary hypertension } \\
\text { and aortic insufficiency }\end{array}$ & 3 & VSD with ruptured sinus of Valsalva & 1 \\
\hline VSD with patent ductus arteriosus & 3 & \multirow{2}{*}{$\begin{array}{l}\text { VSD with atrial septal defect, } \\
\text { infundibular stenosis and valvular } \\
\text { pulmonic stenosis }\end{array}$} & \multirow[t]{2}{*}{1} \\
\hline VSD with aortic insufficiency & 2 & & \\
\hline \multirow[t]{2}{*}{$\begin{array}{l}\text { VSD with infundibular stenosis } \\
\text { and aortic insufficiency }\end{array}$} & \multirow[t]{2}{*}{1} & $\begin{array}{l}\text { VSD with patent ductus arteriosus } \\
\text { and valvular pulmonic stenosis }\end{array}$ & 1 \\
\hline & & $\begin{array}{l}\text { VSD with atrial septal defect and } \\
\text { infundibular stenosis }\end{array}$ & 1 \\
\hline
\end{tabular}

VSD = Ventricular septal defect

TABLE II. Classification of Cardiac Lesions (II)

\begin{tabular}{c|c|c|c}
\hline $\begin{array}{l}\text { Iso- } \\
\text { lated } \\
\text { VSD }\end{array}$ & $\begin{array}{l}\text { VSD with infundibular } \\
\text { stenosis and/or valvular } \\
\text { pulmonic stenosis }\end{array}$ & $\begin{array}{l}\text { VSD with pulmonary } \\
\text { hypertension with or } \\
\text { without associated cardio- } \\
\text { vascular anomalies }\end{array}$ & $\begin{array}{l}\text { VSD with associated } \\
\text { cardiovascular anomalies } \\
\text { without pulmonary } \\
\text { hypertension }\end{array}$ \\
\hline 52 & 20 & 12 & 6
\end{tabular}

groups from their hemodynamic patterns in this paper (Table II). Sixteen lead electrocardiograms were taken in most instances.

A bubble oxygenator of the DeWall type was used in each instance with flow rates calculated at approximately $50 \mathrm{ml}$. per $\mathrm{kg}$. of body weight. The perfusion time ranged from 10.5 to $55 \mathrm{~min}$. and averaged 23 minutes. No left ventricular cardiotomies were done. Induced cardiac arrest by potassium citrate was used in 26 patients, including 4 who had infundibular stenosis as well as ventricular septal defects. These patients have been followed from 6 weeks to 29 months after operation. The electrocardiograms were taken at return visits 1 to 3 months after operation and at later re-examination if significant electrocardiographic changes were found earlier. The changes in the precordial leads were mainly studied in this paper from the purpose mentioned above.

An rS complex is defined here as initial positive deflection of less than 0.8 $\mathrm{mv}$. in amplitude and smaller than the associated $S$ wave in patients up to 8 years of age, of less than $0.7 \mathrm{mv}$. and smaller than the $\mathrm{S}$ wave in patients from 9 to 12 
years of age, of less than $0.6 \mathrm{mv}$. in amplitude and smaller than the $\mathrm{S}$ wave in patients 13 to 16 years of age and of less than $0.5 \mathrm{mv}$. in amplitude and smaller than the $\mathrm{S}$ wave in adults. Some of the electrocardiograms with RS complexes in lead $V_{1}$ were interpreted as right ventricular hypertrophy depending on the ratio of the $\mathrm{R}: \mathrm{S}$ and ages of the patients. ${ }^{5}$ Monophasic $\mathrm{R}$ waves typical of right ventricular hypertorphy were uncommon except in patients with a severe Tetralogy of Fallot. The tracings with $\mathrm{rSr}^{\prime}$ complexes were not interpreted as showing right bundle branch block. Instead, I believe the r' deflection results from the electrical position or from degrees of hypertrophy in the region of the right ventricular outflow tract (the so-called crista pattern). 316)78/9/10) Tracings with $\mathrm{rSR}^{\prime}$ complexes in lead $\mathrm{V}_{1}$ were interpreted as either right bundle branch block or right ventricular hypertrophy depending upon the duration of the initial $r$ wave and the amplitude and duration of the secondary $R$ wave, according to the criteria of Myers. ${ }^{11}$ An $\mathrm{rSR}^{\prime}$ configuration in the right ventricular leads with abnormal prolongation of the initial $r$ wave but with increased amplitude and duration of the secondary $R$ wave has been interpreted as evidence of right ventricular hypertrophy rather than bundle branch block.

The polarity and normal range in the amplitude of $\mathrm{T}$ wave were referred to the values obtained by Ziegler.121

\section{RESULTS}

Marked regression of the amplitude and duration of $\mathrm{R}$ or $\mathrm{R}^{\prime}$ wave in the right ventricular leads after surgery occurred in 7 of the patients and is consistent with such a view point (Fig. la and 1b). Table III shows their electrocardiographic data and interpretations. Incomplete right bundle branch block in 3 patients disappeared after operation. Right ventricular hypertrophy in 4 patients also disappeared in 3 and regressed in degree in the remaining one. In addition, 14 of the patients recorded not marked but definite regression of the amplitude of $\mathrm{R}$ wave in the right ventricular leads and the morphological alteration from $\mathrm{RS}$ to rS pattern. The electrocardiographic data and interpretations were given in Table IV. The electrocardiograms were interpreted as normal in 6 of 7 patients before and after operation. All but 2 showed the changes from RS to $\mathrm{SS}$ complex in the morphological appearance after surgery.

These regressions toward normal have been overshadowed by the more frequent appearance of obvious conduction disturbances in the right ventricular leads which can be interpreted only as bundle branch block (Table V). Some of these have been bizarre as seen in Fig. 2. Table VI shows incomplete right bundle branch block and complete right bundle branch block were equally frequent regardless the preoperative electrocardiograms, except in 7 patients who developed complete right bundle branch block from incomplete right bundle branch block. No specific relations could be elicited between the preoperative electro- 

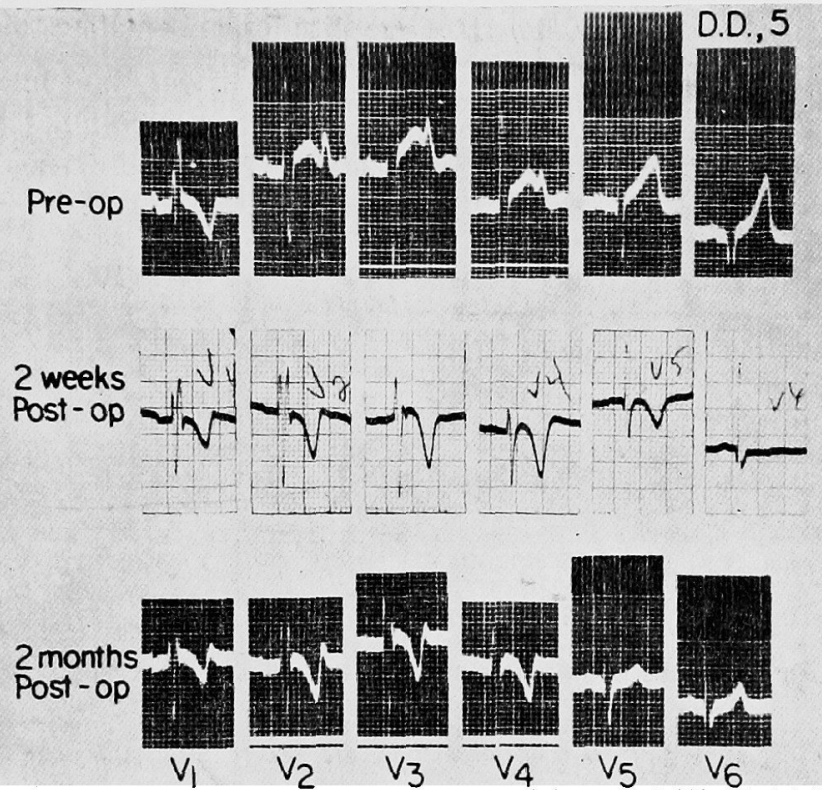

Fig. la. 5 year-old boy with ventricular septal defect and infundidular stenosis.

Lead $V_{1}$ shows regression of the changes of incomplete right bundle branch block.

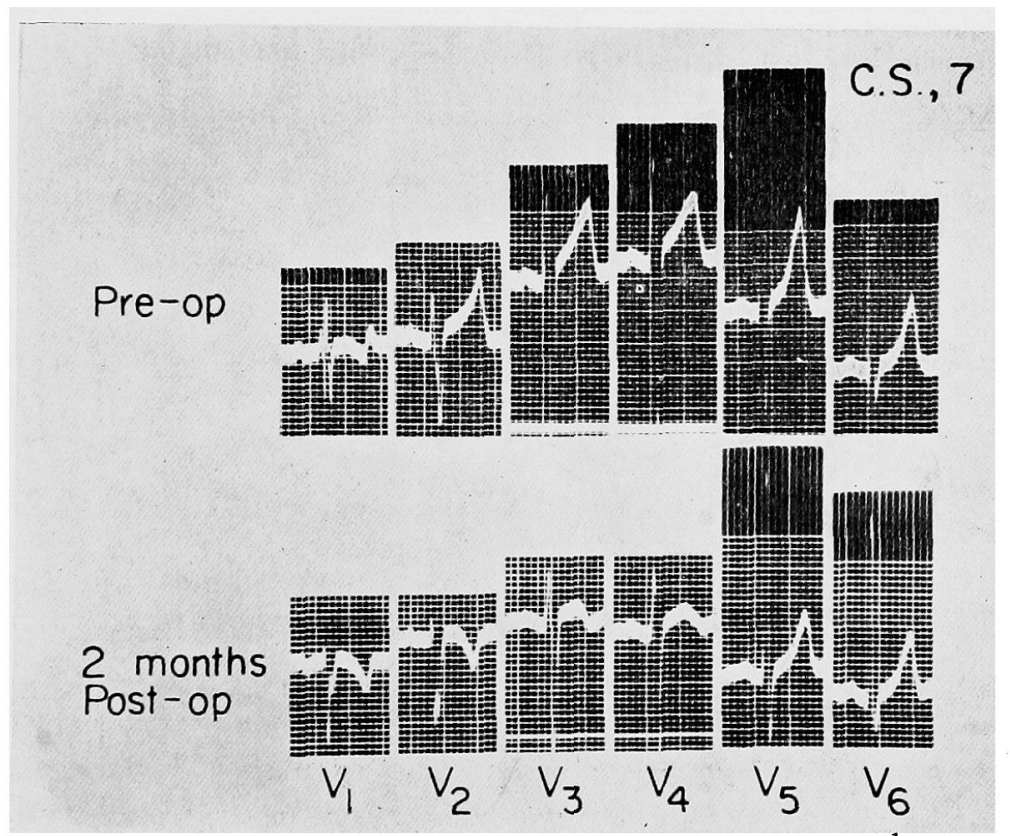

Fig. 1b. 7 year-old girl with 2 ventricular septal defects. The secondary $R$ wave in $V_{1}$ disappeared after operation. 
TABLE III. Electrocardiographic Data (I)

\begin{tabular}{|c|c|c|c|c|c|c|c|c|c|c|c|}
\hline Age & Diagnosis & & $\begin{array}{l}\text { QRS } \\
\text { pat- } \\
\text { tern }\end{array}$ & $\begin{array}{c}\text { QRS } \\
\text { dura- } \\
\text { tion }\end{array}$ & $\begin{array}{l}\text { Intrinsi- } \\
\text { coid } \\
\text { deflection }\end{array}$ & ${ }^{\mathrm{R}} \mathrm{V}_{1}$ & $\mathrm{~S}_{\mathrm{I}}$ & $\stackrel{R}{V_{6}}$ & $\mathrm{~S}_{6}$ & $\mid \begin{array}{c}\text { Interval } \\
\text { after } \\
\text { opera- } \\
\text { tion (Mo.) }\end{array}$ & $\begin{array}{l}\text { Interpre- } \\
\text { tation }\end{array}$ \\
\hline 7 & $\begin{array}{l}\text { VSD } \\
\text { (2 defects) }\end{array}$ & $\begin{array}{l}\mathrm{B} \\
\mathrm{A}\end{array}$ & $\begin{array}{r}\mathrm{rSR}^{\prime} \\
\mathrm{rS}\end{array}$ & $\begin{array}{l}0.08 \\
0.08\end{array}$ & $\begin{aligned} r & =0.02 \\
R^{\prime} & =0.07 \\
r & =0.02\end{aligned}$ & $\begin{array}{l}7 \\
1\end{array}$ & $\begin{array}{l}6 \\
9\end{array}$ & $\begin{array}{l}33 \\
31\end{array}$ & $\begin{array}{l}15 \\
10\end{array}$ & 2 & $\begin{array}{l}\text { RBBB inc. } \\
\text { Normal }\end{array}$ \\
\hline 5 & $\begin{array}{l}\text { VSD with } \\
\text { inf. } \\
\text { stenosis }\end{array}$ & $\begin{array}{l}\mathrm{B} \\
\mathrm{A}\end{array}$ & $\left|\begin{array}{c}\mathrm{rSR} \\
\mathrm{rSr}^{\prime}\end{array}\right|$ & $\begin{array}{l}0.08 \\
0.08\end{array}$ & $\begin{aligned} \mathrm{r} & =0.02 \\
\mathrm{R}^{\prime} & =0.07 \\
\mathrm{r} & =0.02 \\
\mathrm{r}^{\prime} & =0.06\end{aligned}$ & $\begin{array}{r}10 \\
3\end{array}$ & 2 & 19 & 2 & 2 & $\begin{array}{l}\text { RBBB inc. } \\
\text { Normal }\end{array}$ \\
\hline 9 & VSD & $\begin{array}{l}\mathrm{B} \\
\mathrm{A}\end{array}$ & $\mid \begin{array}{l}\mathrm{rSR}^{\prime} \\
\mathrm{rSR}\end{array}$ & $\begin{array}{l}0.09 \\
0.08\end{array}$ & $\begin{array}{l}\mathrm{R}^{\prime}=0.07 \\
\mathrm{R}^{\prime}=0.05\end{array}$ & $\begin{array}{l}18.5 \\
10.5\end{array}$ & $\begin{array}{r}5 \\
12\end{array}$ & $\begin{array}{l}41.5 \\
24\end{array}$ & $\begin{array}{l}8 \\
5.5\end{array}$ & 3 & $\begin{array}{l}\text { RBBB ino. } \\
\text { Normal }\end{array}$ \\
\hline 5 & $\begin{array}{l}\text { VSD with } \\
\text { inf. } \\
\text { stenosis }\end{array}$ & $\begin{array}{l}B \\
A\end{array}$ & $\begin{array}{l}\mathrm{R} \\
\mathrm{rS}\end{array}$ & $\begin{array}{l}0.07 \\
0.07\end{array}$ & 0.04 & $\begin{array}{l}8 \\
3\end{array}$ & $\begin{array}{l}0 \\
3\end{array}$ & $\begin{array}{l}17 \\
25\end{array}$ & $\begin{array}{l}6 \\
0\end{array}$ & 2 & $\begin{array}{l}\text { RVH } \\
\text { Normal }\end{array}$ \\
\hline 9 & $\begin{array}{l}\text { VSD with } \\
\text { PH }\end{array}$ & $\begin{array}{l}\mathrm{B} \\
\mathrm{A}\end{array}$ & $\begin{array}{r}\mathrm{RS} \\
\mathrm{rS}\end{array}$ & $\begin{array}{l}0.09 \\
0.08\end{array}$ & $\begin{aligned} R & =0.02 \\
r & =0.02\end{aligned}$ & $\begin{array}{l}6 \\
2\end{array}$ & $\begin{array}{r}25 \\
8\end{array}$ & $\begin{array}{r}4 \\
14\end{array}$ & $\begin{array}{l}11 \\
17\end{array}$ & 5 & $\begin{array}{l}\text { RVH } \\
\text { Normal }\end{array}$ \\
\hline 3 & VSD & B & $\begin{array}{l}\mathrm{rSR} \\
\mathrm{rSR}\end{array}$ & $\begin{array}{l}0.06 \\
0.07\end{array}$ & $\begin{array}{l}R^{\prime}=0.03 \\
R^{\prime}=0.02\end{array}$ & $\begin{array}{c}12.5 \\
9\end{array}$ & $\begin{array}{r}6 \\
15\end{array}$ & $\begin{array}{l}19 \\
28\end{array}$ & $\begin{array}{l}7 \\
9\end{array}$ & 2 & $\begin{array}{l}\text { RVH } \\
\text { Normal }\end{array}$ \\
\hline 8 & $\begin{array}{l}\text { VSD with } \\
\text { inf. } \\
\text { stenosis }\end{array}$ & $\begin{array}{l}\mathrm{B} \\
\mathrm{A}\end{array}$ & $\begin{array}{l}\mathrm{R} \\
\mathrm{R}\end{array}$ & $\begin{array}{l}0.07 \\
0.09\end{array}$ & $\begin{array}{l}R=0.035 \\
R=0.04\end{array}$ & $\begin{array}{l}44 \\
15\end{array}$ & 0 & $\begin{array}{l}24 \\
24\end{array}$ & $\begin{array}{r}13 \\
9\end{array}$ & 2 & $\begin{array}{l}\text { RVH } \\
\text { RVH }\end{array}$ \\
\hline
\end{tabular}

$\mathrm{B}=$ Before operation $\mathrm{A}=$ After operation $\quad \mathrm{VSD}=$ Ventricular septal defect $\mathrm{PH}=$ Pulmonary hypertention Inf. st. $=$ Infundibular stenosis $\mathrm{RBBB}=$ Right bundle branch block $\mathrm{RVH}=$ Right ventricular hypertrophy
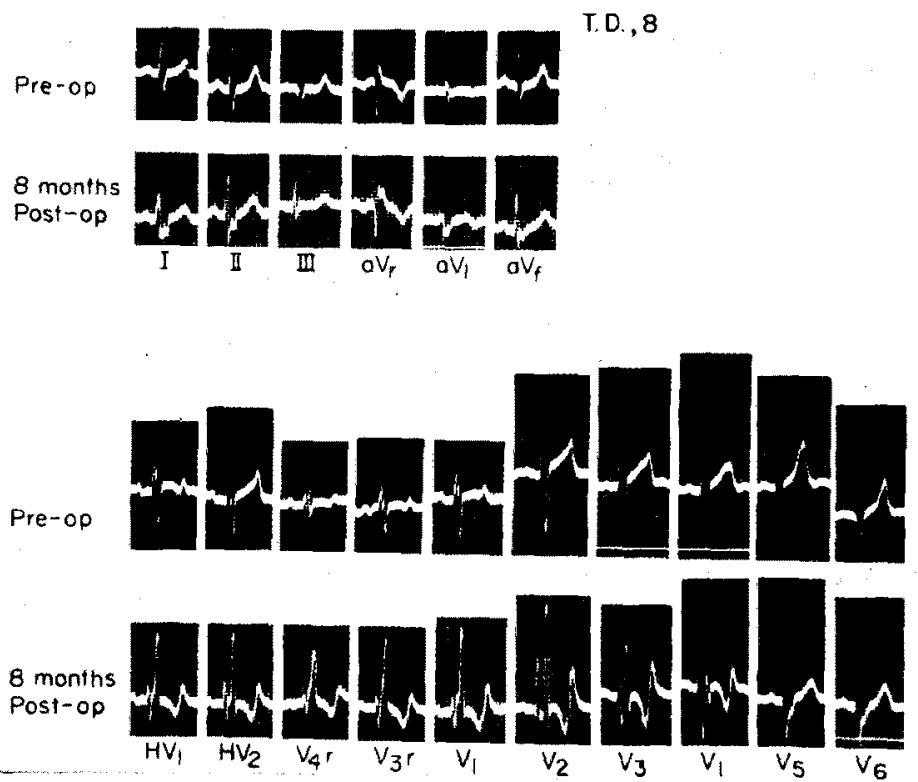

Fig. 2. 8 year-old boy with isolated ventricular septal defect.

Complete right bundle branch block with a QRS duration of 0.16 appeared after operation. 
TABLE IV. Electrocardiographic Data (II)

\begin{tabular}{|c|c|c|c|c|c|c|c|c|c|c|c|}
\hline Age & Diagnosis & & $\begin{array}{l}\text { QRS } \\
\text { pat- } \\
\text { tern }\end{array}$ & $\mid \begin{array}{c}Q R S \\
\text { dura- } \\
\text { tion }\end{array}$ & $\begin{array}{c}\text { Intrinsi- } \\
\text { coid } \\
\text { deflection }\end{array}$ & $\mathrm{R}_{V_{1}}$ & $\mathrm{~s}_{1}$ & $\stackrel{\mathbf{R}_{V_{6}}}{ }$ & $\mathrm{~S}_{6}$ & $\left|\begin{array}{c}\text { Interval } \\
\text { after } \\
\text { opera- } \\
\text { tion (MO.) }\end{array}\right|$ & $\begin{array}{l}\text { Interpre- } \\
\text { tation }\end{array}$ \\
\hline 6 & VSD & $\begin{array}{l}\mathrm{B} \\
\mathrm{A}\end{array}$ & $\begin{array}{l}\mathrm{R} \\
\mathrm{R}\end{array}$ & $\begin{array}{l}0.09 \\
0.10\end{array}$ & $\begin{array}{l}0.06 \\
0.07\end{array}$ & $\begin{array}{r}15 \\
8\end{array}$ & $\begin{array}{l}0 \\
0\end{array}$ & $\begin{array}{l}8 \\
8.5\end{array}$ & $\begin{array}{c}11 \\
5.5\end{array}$ & 1 & $\begin{array}{l}\text { RVH } \\
\text { RVH }\end{array}$ \\
\hline 11 & VSD & $\begin{array}{l}\mathrm{B} \\
\mathrm{A}\end{array}$ & $\begin{array}{l}\mathrm{RS} \\
\mathrm{RS}\end{array}$ & $\begin{array}{l}0.07 \\
0.09\end{array}$ & $\begin{array}{l}0.02 \\
0.02\end{array}$ & $\begin{array}{l}18 \\
11\end{array}$ & $\begin{array}{l}23 \\
19\end{array}$ & $\begin{array}{l}27 \\
34\end{array}$ & $\begin{array}{l}0 \\
0\end{array}$ & 6 & $\begin{array}{l}\text { Normal } \\
\text { Normal }\end{array}$ \\
\hline 3 & VSD & $\begin{array}{c}\text { B } \\
\text { A }\end{array}$ & $\begin{array}{r}\mathrm{RS} \\
\mathrm{rS}\end{array}$ & $\begin{array}{l}0.07 \\
0.07 \\
\end{array}$ & $\begin{array}{l}0.02 \\
0.02 \\
\end{array}$ & $\begin{array}{l}8.5 \\
3 \\
\end{array}$ & $\begin{array}{l}26.5 \\
16\end{array}$ & $\begin{array}{l}12 \\
20\end{array}$ & $\begin{array}{l}2 \\
3 \\
\end{array}$ & 2 & $\begin{array}{l}\text { Normal } \\
\text { Normal }\end{array}$ \\
\hline 3 & VSD & $\begin{array}{l}\mathrm{B} \\
\mathrm{A}\end{array}$ & $\underset{\mathrm{rSS}}{\mathrm{RS}}$ & $\begin{array}{l}0.07 \\
0.07\end{array}$ & $\begin{array}{l}0.02 \\
0.02\end{array}$ & $\begin{array}{l}8.5 \\
3\end{array}$ & $\begin{array}{r}13 \\
6\end{array}$ & $\begin{array}{l}24 \\
15\end{array}$ & $\begin{array}{l}3 \\
0.5\end{array}$ & 1.5 & $\begin{array}{l}\text { Normal } \\
\text { Normal }\end{array}$ \\
\hline 11 & $\mathrm{VSD}$ & $\begin{array}{l}\mathrm{B} \\
\mathrm{A}\end{array}$ & $\underset{\mathrm{rS}}{\mathrm{RS}}$ & $\begin{array}{l}0.08 \\
0.08\end{array}$ & $\begin{array}{l}0.02 \\
0.02\end{array}$ & $\begin{array}{l}9 \\
4\end{array}$ & $\begin{array}{r}13 \\
5\end{array}$ & $\begin{array}{l}21 \\
16\end{array}$ & $\begin{array}{l}1.5 \\
1.5\end{array}$ & 3 & $\begin{array}{l}\text { Normal } \\
\text { Normal }\end{array}$ \\
\hline 4 & VSD & $\begin{array}{l}\mathrm{B} \\
\mathrm{A}\end{array}$ & $\underset{\mathrm{rS}}{\mathrm{RS}}$ & $\begin{array}{l}0.08 \\
0.09\end{array}$ & $\begin{array}{l}0.02 \\
0.02\end{array}$ & $\begin{array}{r}10 \\
4\end{array}$ & $\begin{array}{l}17 \\
13\end{array}$ & $\begin{array}{l}16 \\
12\end{array}$ & $\begin{array}{l}3 \\
4\end{array}$ & 7 & $\begin{array}{l}\text { Normal } \\
\text { Normal }\end{array}$ \\
\hline 7 & VSD & $\begin{array}{l}\mathrm{B} \\
\mathrm{A}\end{array}$ & $\begin{array}{c}\mathrm{RS} \\
\mathrm{rS}\end{array}$ & $\begin{array}{l}0.08 \\
0.08\end{array}$ & $\begin{array}{l}0.02 \\
0.02\end{array}$ & $\begin{array}{l}9 \\
1.5 \\
\end{array}$ & $\begin{array}{r}10 \\
7\end{array}$ & $\begin{array}{l}16 \\
22\end{array}$ & $\begin{array}{l}4 \\
9\end{array}$ & 2 & $\begin{array}{l}\text { Normal } \\
\text { Normal } \\
\end{array}$ \\
\hline 3 & VSD & $\begin{array}{l}\mathrm{B} \\
\mathrm{A}\end{array}$ & $\underset{\mathrm{rSS}}{\mathrm{RS}}$ & $\begin{array}{l}0.07 \\
0.06\end{array}$ & $\begin{array}{l}0.02 \\
0.02 \\
\end{array}$ & $\begin{array}{l}8 \\
1 \\
\end{array}$ & $\begin{array}{r}17 \\
7\end{array}$ & $\begin{array}{l}22 \\
24\end{array}$ & $\begin{array}{l}4 \\
6 \\
\end{array}$ & 4 & $\begin{array}{l}\text { Normal } \\
\text { Normal }\end{array}$ \\
\hline 19 & VSD & $\begin{array}{l}\mathrm{B} \\
\mathrm{A}\end{array}$ & $\underset{r S}{\mathrm{RS}}$ & $\begin{array}{l}0.08 \\
0.08\end{array}$ & $\begin{array}{l}0.02 \\
0.02\end{array}$ & $\begin{array}{l}7 \\
2 \\
\end{array}$ & $\begin{array}{r}21 \\
9\end{array}$ & $\begin{array}{r}9 \\
11 \\
\end{array}$ & $\begin{array}{l}1.5 \\
5\end{array}$ & 3 & $\begin{array}{l}\text { Normal } \\
\text { Normal }\end{array}$ \\
\hline 3 & VSD & $\begin{array}{l}\mathrm{B} \\
\mathrm{A}\end{array}$ & $\begin{array}{r}\mathrm{RS} \\
\mathrm{rS}\end{array}$ & $\begin{array}{l}0.07 \\
0.06 \\
\end{array}$ & $\begin{array}{l}0.02 \\
0.02 \\
\end{array}$ & $\begin{array}{l}8 \\
1\end{array}$ & $\begin{array}{l}11 \\
11 \\
\end{array}$ & $\begin{array}{l}24 \\
18 \\
\end{array}$ & $\begin{array}{l}3 \\
1\end{array}$ & 2 & $\begin{array}{l}\text { Normal } \\
\text { Normal }\end{array}$ \\
\hline 5 & VSD & $\begin{array}{l}\mathrm{B} \\
\mathrm{A}\end{array}$ & $\begin{array}{r}\mathrm{RS} \\
\mathrm{rS}\end{array}$ & $\begin{array}{l}0.08 \\
0.08\end{array}$ & $\begin{array}{l}0.03 \\
0.02 \\
\end{array}$ & $\begin{array}{l}8 \\
3 \\
\end{array}$ & 11 & $\begin{array}{l}20 \\
15 \\
\end{array}$ & $\begin{array}{l}5 \\
1\end{array}$ & 4 & $\begin{array}{l}\text { Normal } \\
\text { Normal }\end{array}$ \\
\hline 36 & $\begin{array}{l}\text { VSD, ASD, } \\
\text { PS, Inf. st. }\end{array}$ & $\begin{array}{l}\mathrm{B} \\
\mathrm{A} \\
\end{array}$ & $\begin{array}{r}\mathrm{RSS} \\
\mathrm{rSS}\end{array}$ & $\begin{array}{l}0.08 \\
0.08\end{array}$ & $\begin{array}{l}0.02 \\
0.02 \\
\end{array}$ & $\stackrel{5}{2}$ & $\begin{array}{l}11 \\
16\end{array}$ & $\begin{array}{r}8 \\
13\end{array}$ & $\begin{array}{l}5 \\
3\end{array}$ & 3 & $\begin{array}{l}\text { Normal } \\
\text { Normal }\end{array}$ \\
\hline 6 & $\begin{array}{l}\mathrm{VSD} \\
\mathrm{AI}\end{array}$ & $\begin{array}{l}\mathrm{B} \\
\mathrm{A}\end{array}$ & $\underset{\mathrm{rSS}}{\mathrm{RS}}$ & $\begin{array}{l}0.07 \\
0.07\end{array}$ & $\begin{array}{l}0.02 \\
0.02\end{array}$ & $\begin{array}{l}8 \\
3\end{array}$ & $\begin{array}{l}12 \\
14\end{array}$ & $\begin{array}{l}29 \\
35\end{array}$ & $\begin{array}{l}0 \\
0\end{array}$ & 1.5 & $\begin{array}{l}\text { Normal } \\
\text { Normal }\end{array}$ \\
\hline 20 & $\begin{array}{l}\text { VSD } \\
\mathrm{PH}\end{array}$ & $\begin{array}{l}B \\
A\end{array}$ & $\underset{\mathrm{rS}}{\mathrm{R}}$ & $\begin{array}{l}0.10 \\
0.10\end{array}$ & $\begin{array}{l}0.02 \\
0.02\end{array}$ & $\begin{array}{l}8 \\
4\end{array}$ & $\begin{array}{r}0 \\
19\end{array}$ & $\begin{array}{l}38 \\
26\end{array}$ & $\begin{array}{l}5 \\
5\end{array}$ & 12 & $\begin{array}{l}\text { Normal } \\
\text { Normal }\end{array}$ \\
\hline
\end{tabular}

$B=$ Before operation $\quad A=$ After operation $\quad V S D=$ Ventricular septal defect $A S D=$ Atrial septal defect $\quad A I=$ Aortic insufficiency $\quad$ Inf. st. $=$ Infundibular stenosis $\quad \mathrm{PS}=\mathrm{Pulmonic}$ stenosis $\quad \mathrm{PH}=$ =Pulmonary hypertension $\quad \mathrm{RVH}=$ Right ventricular hypertrophy

cardiograms and postoperative occurrence of right bundle branch block. Of 80 patients who had no conduction distrubances before operation, 41 patients or $51.2 \%$ developed obvious right bundle branch block after operation. Table $\mathrm{V}$ and VI also indicate that incomplete and complete right bundle branch block were equally frequent in the first group (with isolated ventricular septal defect) and in the third group (with ventricular septal defect associated with pulmonary 
TABLE V. Electrocardiographic Interpretation before and after Surgery

\begin{tabular}{|c|c|c|c|c|c|c|}
\hline & & Normal & RVH & IRBBB & CRBBB & Total \\
\hline VSD isolated & $\begin{array}{l}\mathrm{B} \\
\mathrm{A}\end{array}$ & $\begin{array}{l}40 \\
31\end{array}$ & $\begin{array}{l}2 \\
1\end{array}$ & $\begin{array}{r}9 \\
12\end{array}$ & $\begin{array}{l}1 \\
8\end{array}$ & 52 \\
\hline $\begin{array}{l}\text { VSD, infundibular stenosis and/or } \\
\text { valvular pulmonic stenosis }\end{array}$ & $\begin{array}{l}\mathrm{B} \\
\mathrm{A}\end{array}$ & $\begin{array}{l}6 \\
3\end{array}$ & $\begin{array}{r}11 \\
3\end{array}$ & $\begin{array}{l}3 \\
4\end{array}$ & $\begin{array}{r}1 \\
12\end{array}$ & 21 \\
\hline $\begin{array}{l}\text { VSD, pulmonary hypertension } \\
\text { with or without associated } \\
\text { cardiovascular anomalies }\end{array}$ & B & $\begin{array}{l}4 \\
3\end{array}$ & 5 & $\begin{array}{l}4 \\
5\end{array}$ & 0 & 13 \\
\hline $\begin{array}{l}\text { VSD with associated cardiovas- } \\
\text { cular anomalies without } \\
\text { pulmonary hypetension }\end{array}$ & B & $\begin{array}{l}5 \\
2\end{array}$ & $\begin{array}{l}0 \\
0\end{array}$ & $\begin{array}{l}1 \\
1\end{array}$ & $\begin{array}{l}0 \\
3\end{array}$ & 6 \\
\hline Total & $\begin{array}{l}\mathrm{B} \\
\mathrm{A}\end{array}$ & $\begin{array}{l}55 \\
39\end{array}$ & $\begin{array}{r}18 \\
5\end{array}$ & $\begin{array}{l}17 \\
22\end{array}$ & $\begin{array}{r}2 \\
27\end{array}$ & 92 \\
\hline
\end{tabular}

$\mathrm{RVH}=$ Right ventricular hypertrophy

IRBBB $=$ Incomplete right bundle branch block $\quad$ CRBBB $=$ Complete right bundle branch block

TABLE VI. Conduction Disturbances after Operation

\begin{tabular}{|c|c|c|c|c|c|c|}
\hline & $\begin{array}{l}\mathrm{B} \\
\mathrm{A}\end{array}$ & $\begin{array}{l}\text { Normal } \\
\text { IRBBB }\end{array}$ & $\mid \begin{array}{l}\text { Normal } \\
\text { CRBBB }\end{array}$ & $\begin{array}{l}\text { IRBBB } \\
\text { CRBBB }\end{array}$ & $\left|\begin{array}{c}\mathrm{RVH} \\
\mathrm{LRBBB}\end{array}\right|$ & $\begin{array}{l}\text { RVH } \\
\text { CRBBB }\end{array}$ \\
\hline VSD isolated & & 7 & 4 & 3 & 1 & 0 \\
\hline $\begin{array}{l}\text { VSD, infundibular stenosis and/or } \\
\text { valvular pulmonic stenosis }\end{array}$ & & 1 & 4 & 1 & 3 & 6 \\
\hline $\begin{array}{l}\text { VSD, pulmonary hypertension } \\
\text { with or without associated } \\
\text { cardiovascular anomalies }\end{array}$ & & 1 & 1 & 2 & 2 & 1 \\
\hline $\begin{array}{l}\text { VSD with associated cardiovas- } \\
\text { cular anomalies without } \\
\text { pulmonary hypertension }\end{array}$ & & 1 & 2 & 1 & 0 & 0 \\
\hline Total & & 10 & 11 & 7 & 6 & 7 \\
\hline
\end{tabular}

IRBBB = Incomplete right bundle branch block

CRBBB = Complete right bundle branch block $\quad \mathrm{RVH}=$ Right ventricular hypertrophy

hypertension), while complete right bundle branch block appeared most frequently in the second group with ventricular septal defects associated with infundibular stenosis.

Table VII lists the general configurations of the QRS complexes in lead $V_{1}$ before operation and at the last follow-up examination. Frequent appearance of an rSR' pattern and disappearance of an RS pattern after surgery were consistent with the postoperative development of right bundle branch block. These conduction disturbances have persisted relatively unchanged.

Not a single instance developed left bundle branch block. 
TABLE VII. Morphology of QRS Complex in $V_{1}$

\begin{tabular}{|c|c|c|c|c|c|c|c|}
\hline & & $\mathrm{rS}$ & $\mathrm{rSr}^{\prime}$ & $\mathrm{rSR}^{\prime}$ & RS & $\mathrm{R}$ & Total \\
\hline VSD isolated & $\begin{array}{l}\text { B } \\
\text { A }\end{array}$ & $\begin{array}{l}20 \\
25\end{array}$ & $\begin{array}{l}1 \\
4\end{array}$ & $\begin{array}{l}12 \\
19\end{array}$ & $\begin{array}{r}17 \\
2\end{array}$ & $\begin{array}{l}2 \\
2\end{array}$ & 52 \\
\hline $\begin{array}{l}\text { VSD with infundibular stenosis } \\
\text { and/or valvular pulmonic } \\
\text { stenosis }\end{array}$ & $\mathrm{B}$ & $\begin{array}{l}2 \\
2\end{array}$ & $\begin{array}{l}0 \\
1\end{array}$ & $\begin{array}{r}4 \\
14\end{array}$ & $\begin{array}{r}10 \\
0\end{array}$ & 4 & 20 \\
\hline $\begin{array}{l}\text { VSD with pulmonary hypertension } \\
\text { without associated cardiovas- } \\
\text { cular anomalies }\end{array}$ & B & $\begin{array}{l}0 \\
2\end{array}$ & $\begin{array}{l}1 \\
1\end{array}$ & $\begin{array}{l}4 \\
7\end{array}$ & $\begin{array}{l}6 \\
2\end{array}$ & 0 & 12 \\
\hline $\begin{array}{l}\text { VSD with associated cardiovas- } \\
\text { cular anomalies without } \\
\text { pulmonary hypertension }\end{array}$ & $\mathrm{B}$ & 2 & $\begin{array}{l}0 \\
0\end{array}$ & 1 & 4 & 0 & 6 \\
\hline Total & $\begin{array}{l}\text { B } \\
\text { A }\end{array}$ & $\begin{array}{l}23 \\
31\end{array}$ & $\frac{2}{6}$ & $\begin{array}{l}21 \\
44\end{array}$ & $\begin{array}{r}37 \\
4\end{array}$ & $\begin{array}{l}7 \\
5\end{array}$ & 90 \\
\hline
\end{tabular}

TABLE VIII. Polarity of $\mathrm{T}$ Wave and Numbers of Patients

\begin{tabular}{|c|c|c|c|c|c|c|c|c|c|}
\hline & $V_{4.3}$ & $V_{3,2}$ & $V_{1}$ & $V_{2}$ & $\mathrm{~V}_{3}$ & $V_{4}$ & $V_{5}$ & $\mathrm{~V}$ & \\
\hline & $+\quad-$ & $+\quad-$ & - & $+\quad-$ & +- & + & + & + & - \\
\hline B & 882 & $8 \quad 82$ & $25 \quad 65$ & $56 \quad 34$ & $\begin{array}{ll}72 & 18\end{array}$ & $\begin{array}{ll}77 & 13\end{array}$ & $\begin{array}{ll}88 & 2\end{array}$ & 88 & 2 \\
\hline A & $\begin{array}{ll}387\end{array}$ & 288 & 585 & 585 & 1080 & $23 \quad 67$ & $55 \quad 25$ & 81 & 9 \\
\hline
\end{tabular}

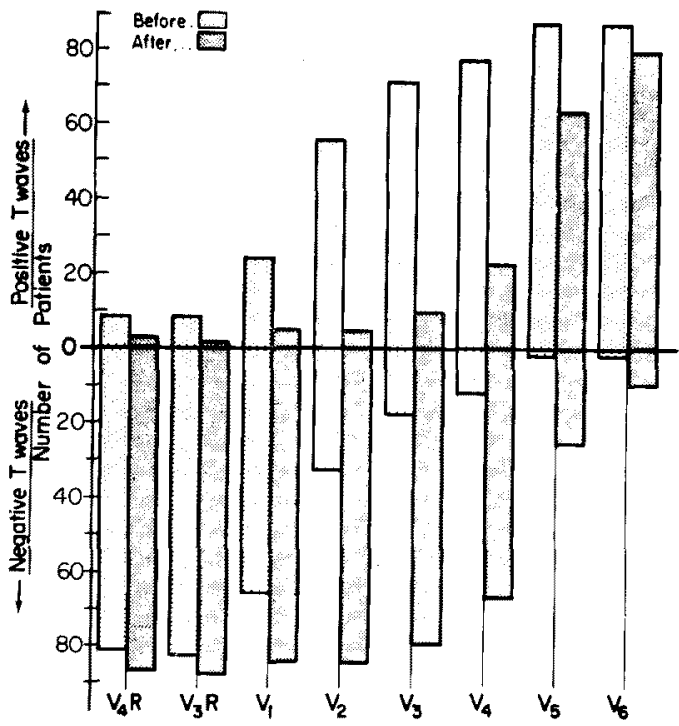

Fig. 3. This tabulation of the polarity of $T$ waves in precordial leads shows the changes which occurred after surgery. A small number of diphasic $\mathrm{T}$ waves are included in the negative $\mathrm{T}$ wave group. 


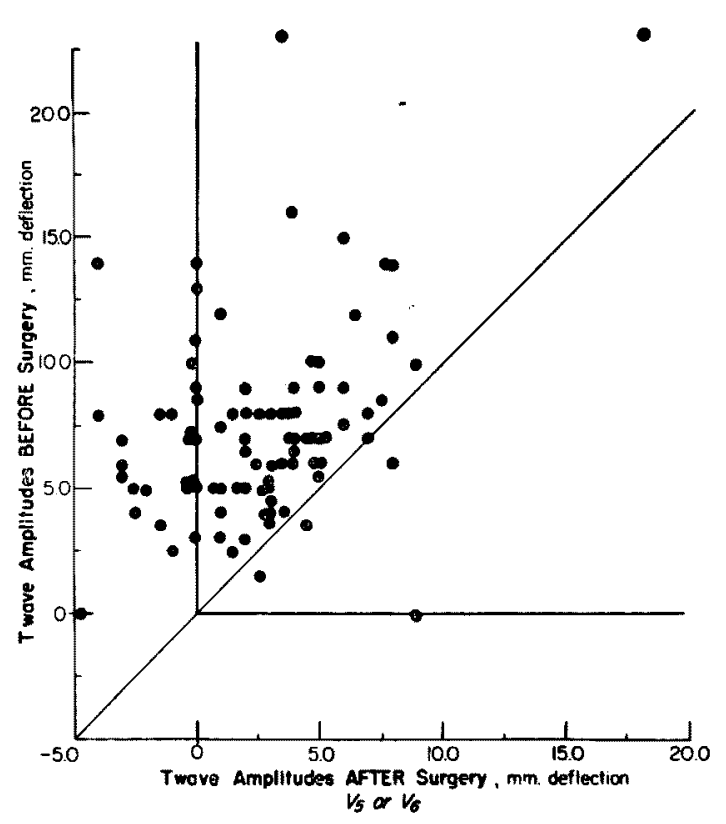

Fig. 4. $T$ wave amplitude in left precordial leads decreased consistently after ventriculotomy.

The ST segment changes in the right ventricular leads after operation occurred in association with the development of right bundle branch block and would not be worthy for estimation of the myocardial changes. The depression of ST segment in the left ventricular leads $\left(\mathrm{V}_{5,6}\right)$ before operation was observed in only 2 patients, who had ventricular septal defect, pulmonary hypertension and aortic insufficiency; ventricular septal defect with ruptured sinus of Valsalva. The ST segment depression in the left ventricular leads occurred in 10 patients after surgery. The ST segment elevation after operation was noted in only one patient, who developed lateral wall infaction due to a coronary air embolism during surgery,

Table VIII and Fig. 3 indicate numbers of patients with the polarity of $\mathrm{T}$ wave in the precordial leads before and after operation. $\mathrm{T}$ wave inversion through $V_{4}$ often is a normal finding in children and, indeed when a positive $\mathrm{T}$ wave occurs in $V_{1}$ in an infant or small child, right ventricular hypertorphy may be present. Most of the patients had positive $\mathrm{T}$ waves in the transition zone and in leads to the left before surgery, but a very large number changed in this respect afterward, particularly in the transition zone. $T$ wave changes extending as far as $V_{5}$ and $V_{6}$ were common, being inverted in $27.7 \%$ or 25 patients in $\mathrm{V}_{5}$ and in $10 \%$ or 9 patients in $\mathrm{V}_{6}$. When positive $\mathrm{T}$ wave persisted in these left ventricular leads, they were decreased in amplitude with exception of 5 in- 
stances (Fig. 4). In Figures 5a and 5b the configuration, depth and extent of $\mathrm{T}$ wave inversion shown are examples of the changes discussed here and can hardly be considered normal.

\section{DISCUSSION}

An $\mathrm{rSR}^{\prime}$ complex in the right ventricular leads interpreted as incomplete right bundle branch block in 3 patients reverted to normal and $41(51 \%)$ of 80 patients who had no evidence of conduction disturbances developed right bundle branch block after repair of ventricular septal defect. How is this procedure responsible for this 2 way postoperative changes? An $\mathrm{rSR}^{\prime}$ pattern - incomplete right bundle branch block - has been known as one of characteristic electrocardiographic features in patients with atrial septal defects, whose hemodynamic situations are somewhat similar to that in patients with ventricular septal detect. 21 patients in this series had also $\mathrm{rSR}^{\prime}$ complexes in the right ventricular leads. No instance developed the postoperative conduction disturbances in patients with atrial septal defects, except in patients with ostium primum. ${ }^{14)}$ And if a block really existed, how did these changes in the appearance occur? How could one explain the disappearance or regression in amplitude of $\mathrm{R}$ or $\mathrm{R}^{\prime}$ after the closure of septal defect? The significance and the cause of $\mathrm{rSR}^{\prime}$ complex in the right ventricular leads in patients with cardiac septal defects will be discussed.

Peñaloza et $a l^{71}$ described that the $\mathrm{R}^{\prime}$ wave represented the vector of the depolarization of the basal portions of the both ventricles and of the higher parts of the interventricular septum. Kossman et $a l .{ }^{8}$ ) and Sodi-Pallares ${ }^{9}$ ) studied the intracardiac electrocardiography and also reached the same conclusion. Investigations of Kjellberg et al. ${ }^{10)}$ showed that in atrial septal defects there is a predominant hypertrophy of the crista supraventricularis and dilatation of the right ventricular chamber at this level. All these previous studies point out that this characteristc right ventricular configuration means anatomic changes in the basal regions of the right ventricle and the higher parts of the interventricular septum.

Rushmer et al. ${ }^{131}$ established that the right ventricular contraction is effected primarily by shortening of the ventricle along its longitudinal axis, with no significant alterations in the width of ventricular cavity. The muscles of the basal region, possibly because of their situation (outflow tract), seems to bear the major burden created by the excessive blood volume, which certainly brings about dilatation and hypertrophy in this region. Prinzmetal ${ }^{15}$ showed that in the basal portions of both ventricles, on account of the diminutive number of fibers of Purkinje as compared with other regions of the heart, the wave of depolarization travels more slowly. Such phenomenon will facilitate in a special way a delay in conduction of these parts dilated and/or hypertrophied. As soon 
as pulmonary hypertension develops, these regions will not be able to cope with the hemodynamic burden, and the whole right ventricle will hypertrophy in order to overcome the increased pulmonary arteriolar resistance. As a consequence of this generalized hypertrophy there is no longer a predominance of the basal regions, so that the electrocardiographic pattern of concentric hypertrophy is superimposed, giving rise to the $\mathrm{R}$ or $\mathrm{RS}$ complex.

Walker et al. ${ }^{11}$ studied the electrocardiographic changes in patients with atrial septal defects before and after repair of the septal defects and tried to establish a correlation between the patterns of QRS complex in the right ventricular leads and the right ventricular pressure, and emphasized that the presence of secondary $\mathrm{R}$ wave was not due to irreversible conduction disturbances, but was rather reversible by correction of atrial septal defects. de Oliveira et al. ${ }^{2)}$ clearly demonstrated that the electrocardiographic morphology was definitely related to hemodynamics, particularly the magnitude of shunt flow, in their correlative study of patients with atrial septal defects, and confirmed that $\mathrm{rSR}^{\prime}$ or $\mathrm{rSR}^{\prime} \mathrm{s}^{\prime}$ complex in lead $V_{1}$, as far as an atrial septal defect is concerned, does not mean conduction distrubances in the right bundle branch of bundle of His, but delay in activation of the basal portions of the right ventricle and of the higher parts of interventricular septum. Most of the patients with atrial septal defect the author has observed developed similar electrocardiographic changes after surgery. ${ }^{14)}$ One of the most striking example was a 48 year-old woman who had atrial septal defect with pulmonary hypertension and complete right bundle branch block with QRS duration of 0.12 but her electrocardiogram showed incomplete right bundle branch block 11 months after operation and was entirely in normal range 27 months after operation. The changes observed in patients with Trilogy of Fallot (atrial septal defect and valvular pulmonic stenosis with intact interventricular septum), in whom monophasic $R$ waves in the right ventricular leads were common and $\mathrm{rSR}^{\prime}$ complexes and gradual regression toward normal developed, can be also explained from the same point of view. ${ }^{14)}$ These changes also indicate that the regression occurs in a completely reversed fashion, described in the appearance of $\mathrm{rSR}^{\prime}$ and $\mathrm{RS}$ or $\mathrm{R}$ complexes.

Ventricular septal defect usually lies in the outflow portion of the right ventricle and this portion of the right ventricle alone may function as a short tract between the left ventricle and the pulmonary artery so that the chamber of the right ventricle is functionally involved to a relatively minor extent. This may be possible cause of relatively less frequent appearance of rSR' complex in patients with ventricular septal defect, but the appearance and regression can be explained from the same basis.

Secondary $R$ wave is also explained to represent diastolic overloading of the right ventricle. ${ }^{\text {b) }}$ This concept is well fit to the electrocardiograms in patients with atrial septal defect as discussed above. The postoperative occurrence of 
right bundle branch block does not agree with this, however, since the rSR' pattern persisted although abnormal hemodynamics had been corrected by surgery. It is now apparent that right bundle branch block does not occur when the interventricular septum is intact.

The appearance of right bundle branch block in high percentage in this study is in marked contrast to the postoperative disappearance or regression of secondary $\mathrm{R}$ wave in $\mathrm{rSR}^{\prime}$ complexes in a majority of patients with atrial septal defect and some of patients with ventricular septal defect. Its occurrence has been mentioned sporadically but the true incidence was not known. Truex et $\left.a l .{ }^{16}\right)$ investigated extensively the location of the cardiac conduction system in relation to defects of the membranous ventricular septum. Of 15 , in 12 specimens, the common bundle as well as the left and right bundle branch were located in the postero-inferior margin of the septal defect. Therefore the right bundle branch may be injured in particular, because of the great variability in both the branching of the common bundle at the postero-inferior angle of the defect and in the course of the right bundle branch in the interventricular septum. The right bundle may descend either posterior or anterior to the defect and in the form of one or several fascicles. Dickens et $a l .{ }^{17}$ ) stand on the same opinion that the postoperative rSR $^{\prime}$ complex in patients with ventricular septal defect represent right bundle branch block caused by injury of the conduction system. They suggested that vectrocardiogram is useful for differentiation between right bundle branch block and right ventricular hypertrophy in $\mathrm{rSR}^{\prime}$ patterns in the right ventricular leads, and presumed that these postoperative electrocardiographic changes are traumatic in origin, resulting from the inadvertent placement of sutures around the defect at surgery. Indeed, the auther still clearly remembers that while witnessing an operation the abnormal conduction was noted to occur during the procedure, as a suture was being placed around the rim of defect and returned to normal when the suture was removed. Similar experiences apparently have been noted by the other groups reporting on the repair of ventricular septal defect by open heart surgery. Kirklin et $a{ }^{18}{ }^{18}$ ) have called attention to the presumed location of the conduction system in the inferior angle of the defect. They also noted that by protecting this area during surgical closure of septal defects in 25 patients, they had encountered no conduction disturbances. It is now clear that the postoperative occurrence of right bundle branch block is just accidental and it can be expected that its occurrence would be less frequent in future by more skilled technique which protects the postero-inferior margins of membranous septal defect from sutures and strong tractions during surgery. And then why is the incidence of right bundle branch block in patients with Tetralogy of Fallot so frequent? Dickens et al. ${ }^{17}$ ? raised the question of possible injury to the conduction tissue during the performance of the pulmonary valvotomy in these patients. Their speculation came from the fact that 


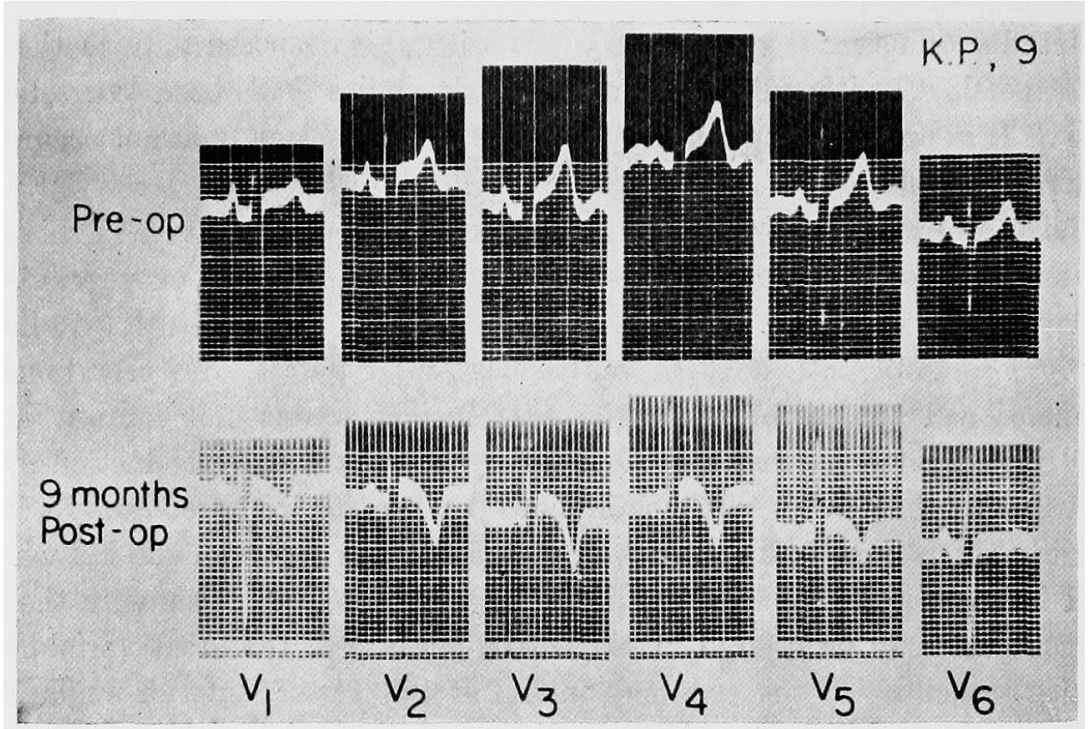

Fig. 5a. 9 year-old girl had ventricular septal defect with pulmonary hypertension.

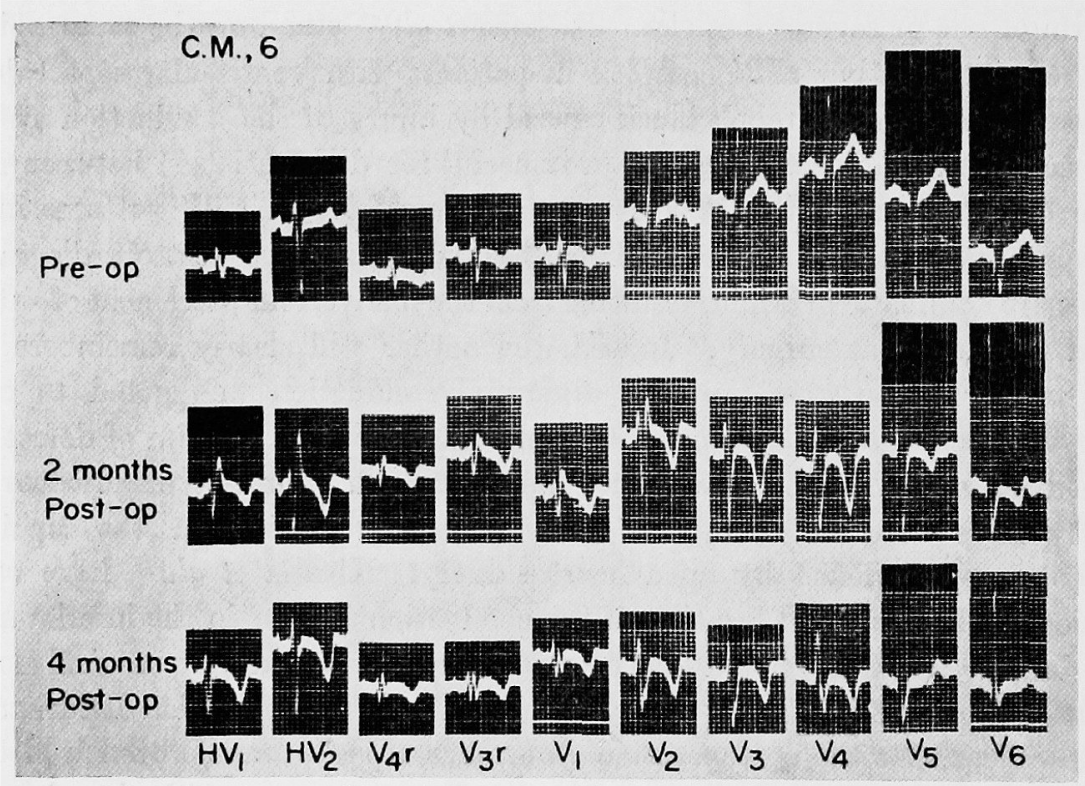

Fig. 5b. 6 year-old girl had ventricular septal defect with pulmonary hypertension.

Significant $\mathrm{T}$ wave changes appeared in comparable leads after ventriculotomy. Patient C.M. has also had recurrent paroxymal atrial flutter since operation. Neither of these patients was on digitalis at the time these tracings were taken. 
periarterial conduction tissue was dissected about a transposed pulmonary artery in one case reported by Truex et al. ${ }^{15)}$ In addition to injury to the conduction tissue hemorrhage within the atrioventricular node and bundle branch was noted at postmortem examination in some of their cases. The exact cause can not be determined at the present time.

$\mathrm{T}$ wave abnormalities in the precordial leads have been common findings after operation in this study and the magnitude of the changes frequently has exceeded that which one might expect from thoracotomy and opening of the pericardium alone (Fig. 5). "Surgical pericarditis" is an unlikely explanation for the $\mathrm{T}$ wave abnormalities, particularly in view of the fact that a large number of patients in my experience who have had atriotomy for the repair of atrial septal defects under hypothermia have not shown persistent $\mathrm{T}$ wave changes. What then are the possible mechanisms for injury to muscle? Trauma to the myocardium from manipulation alone is probably minimal in most of the patients. Cardiac massage and electrical defibrillation are known to produce muscle damage, $\left.{ }^{19}\right)$ but these measures seldom are necessary when the pump oxygenator is used. Even in the mechanically inactive state, the heart continues to con-
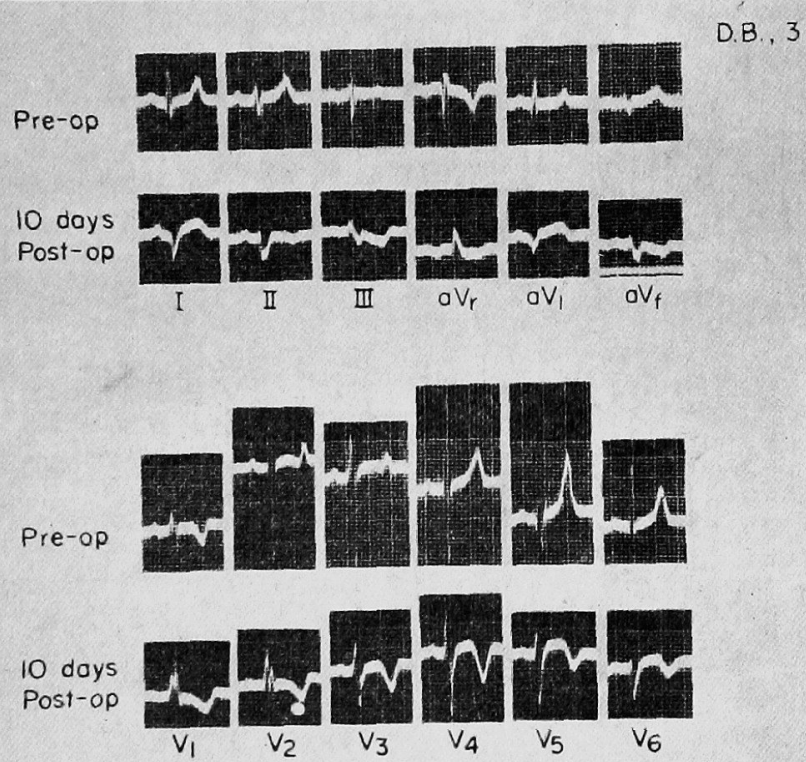

Fig. 6. 13 year-old body with isolated ventricular septal defect.

Coronary air embolism was observed at operation. A short period of ventricular fibrillation followed. Electrocardiographic signs of myocardial infarction developed. $Q$ waves appeared in leads $I$ and AVL. There were significant decrease in $R$ wave amplitude in left precordial leads. 
sume oxygen as long as it is available. Cardiac arrest with prolonged interruption of the blood supply to the heart muscle might therefore produce some degenerative changes even though the muscle is not contracting, but induced cardiac arrest was not a common denominator in the patients who showed some of the most extensive $\mathrm{T}$ wave changes in the precordial leads. Coronary air embolism would seem to be another possible explanation for these changes. It has been observed on several occasions, and usually does not produce permanent damage, but it was associated with the electrocardiographic signs of myocardial infarction in one boy (Fig. 6). Small bubbles in the coronary arteries may be "washed out" of the vascular bed if the perfusion pressure is high enough, but removal by forcing the air on through to the venous side or by diffusion and solution of the gases may not be complete before the injury occurs. A fatal myocardial infaction occurred in one girl due to extension of a thrombus into the left coronary artery (Fig. 7). This thrombus originated in an area of aneurysmal dilatation of the aorta at the site of aortotomy for correction of an unsupported aortic cusp. Rarely aortic insufficiency appearing or increasing after operation on the basis of an unsupported aortic cusp (Fig. 8) accounts for some $\mathrm{T}$ wave abnormalities.

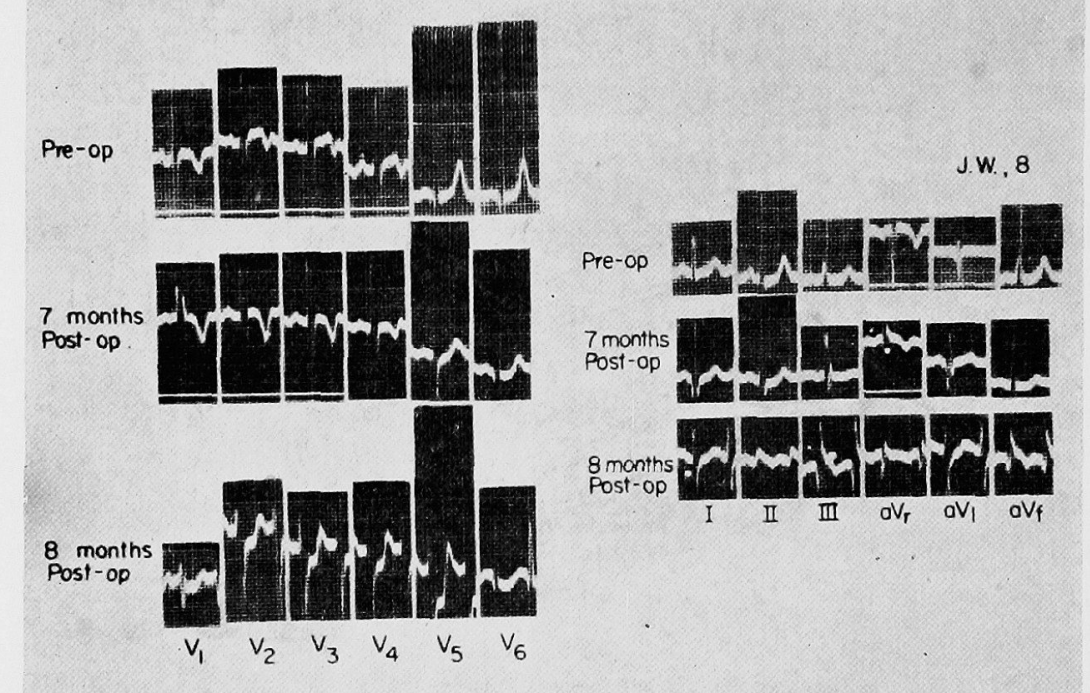

Fig. 7. 8 year-old girl with ventricular septal defect.

Acute posterior myocardial infarction appeared 8 months after operation. An attempt had been made to relieve aortic insufficiency due to prolapse of the cusp adjacent to the defect by creating a bicuspid valve. Aneurysmal dilatation of the aorta developed at the site of the aortotomy. A thrombus formed and propagated into the left coronary artery caused death. 


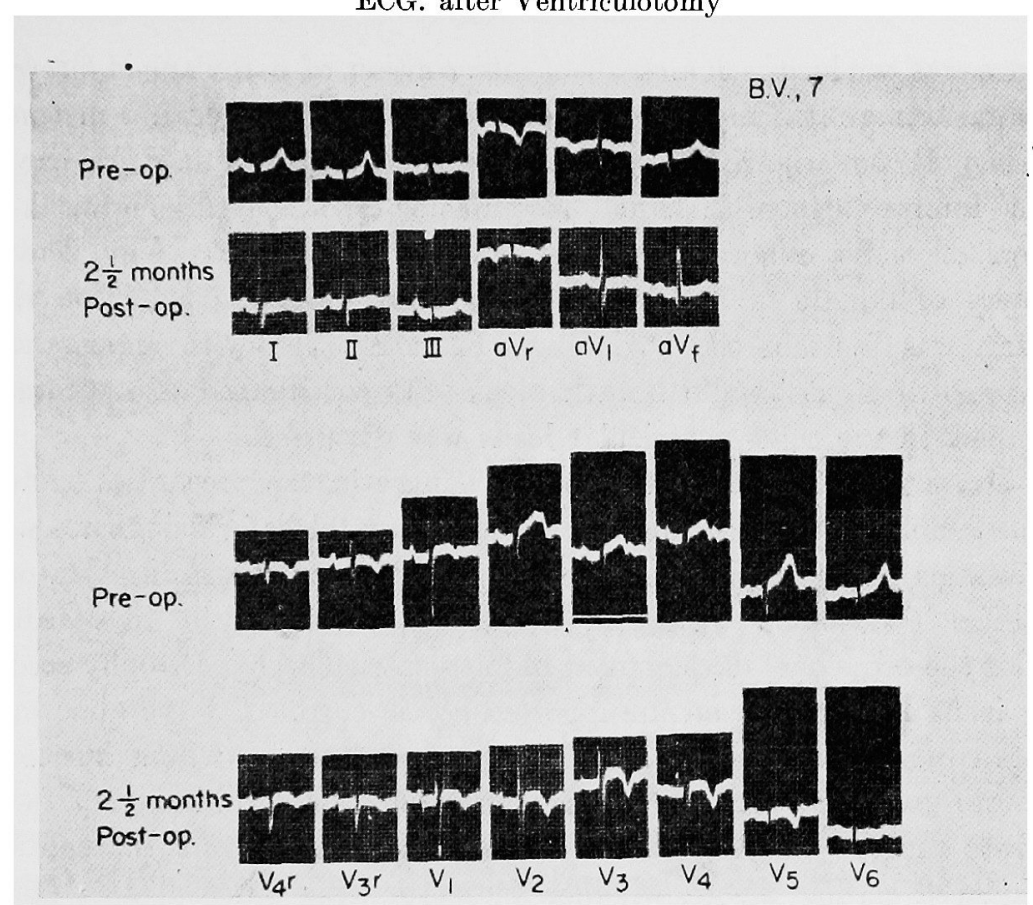

Fig. 8. 7 year-old girl with ventricular septal defect.

This patient had aortic insufficiency because of a prolarsed cusp. The insufficiency increased after repair of the ventricalar defect. This may account for the $\mathrm{T}$ wave changes in left precordial leads.

Myocardial damage from the incision in the free wall or the right ventricle might be associated with abnormalities in direct leads from this area, but would not be expected the widespread changes the author is reporting. Even so, the right ventriculotomy may be crucial to these changes because they are less common in a smaller group of patients whose surgery included the extracorporeal pump but no ventriculotomy. None of the above hypothesis logically account for all of the electrocardiographic abnormalities which have been encountered, and one is left with impression that diffuse muscle damage may occur from ventriculotomy during cardiac by-pass.

\section{SUMMARY}

The electrocardiograms of 90 patients with ventricular septal defects before and after the repair of septal defects were comparatively studied from the standpoint of possible ill effect of open heart surgery on the heart. Right bundle branch block appeared frequently after the closure of ventricular septal defects $(51 \%)$, particularly in those patients who also had infundibular stenosis and in whom some of the infundibular muscle was resected. The magnitude of the postoperative changes of right bundle branch block indicate that a conduction 
disturbance was produced, as a result of interruption of pathways in the septum. These alterations are in contrast to the postoperative disappearance or regression of secondary $\mathrm{R}$ wave in $\mathrm{rSR}^{\prime}$ complex in other patients $(15.5 \%)$ whose preoperative interpretations included incomplete right bundle branch block. Regression of these abnormalities supports the view that right ventricular hypertrophy or bundle branch block rather than delayed conduction through the septum was fault in the beginning. In connection with appearance and disappearance of right bundle branch block, the mechanism of appearance of rSR' complex in the right ventricular leads was discussed.

The appearance of extensive $\mathrm{T}$ wave changes in the precordial leads after right ventriculotomy and closure of ventricular septal defects indicate a significant alteration in ventricular repolarization. The extent and duration of these abnormalities cannot be explained by surgical pericarditis, myocardial infarction, or the occasional development of left ventricular hypertrophy secondary to aortic insufficiency from an unsupported aortic cusp. T wave abnormalities were more common than in a smaller group of patients in whom bubble oxygenator were used for other operations which did not include injury to septal muscle from sutures, coronary air embolism, and trauma from the cardiotomy itself. Induced cardiac arrest, whether anoxic or drug induced, does not have a direct relationship to these changes. The clinical course of these patients thus far indicates that these electrocardiographic changes have no prognostic significance.

\section{References}

I) Walker, W. J. et al., Am. Heart J. 1956, 52, 547.

2) de Oliveira, J. M. et al., Am. Heart J. 1958, 55, 369.

3) Toscano-Barboza, E. et al., Am. J. Card. 1959, 2, 698.

4) Toscano-Barboza, E. et al., Am. J. Card. 1959, 3, 721.

5) Braunwald, E. et al., Am. Heart J. 1955, 50, 591.

6) Cabrera, C. E. et al., Am. Heart J. 1952, 43, 661, 669.

7) Peñaloza, D. et al., Am. Heart J. 1955, 49, 51.

8) Kossman, E. E. et al., Circulation, 1950, 2, 10.

9) Sodi-Pallares, D., New bases of electrocardiography, 1956, C.V. Mosby Co. St. Louis.

10) Kjellberg, S. R. et al., Disagnosis of congenital heart disease, 1955, Year Book Publishers, Inc. Chicago.

11) Myers, G. B., The interpretation of the unipolar electrocardiography, 1956, C.V. Mosby Co. St. Louis.

12) Rushmer, R.F. et al., Circulation Res. 1953, 1, 162.

13) Ziegler, R. F., Electrocardiographic studies in normal infants and children, 1951, Thomas, Springfield, Ill.

14) Sasaki, R., Unpublished data.

15) Prinzmetal, M. et al., 1954, Second World Congress of Cardiology, Washington, D.C.

16) Truex, R.C. et al., J. Thorac. Surg. 1958, 35, 421.

17) Dickens, J. et al., Circulation, 1959, 20, 201.

18) Kirklin, J.W. et al., J. Thorac. Surg. 1957, 33, 45.

19) Peddie, G.H. et al., Surgery, 1956, 40, 481. 